DOI: 10.17516/1997-1397-2021-14-4-497-506

УДК 517.9

\title{
The Problem of Determining of the Source Function and of the Leading Coefficient in the Many-dimensional Semilinear Parabolic Equation
}

\author{
Svetlana V. Polyntseva* \\ Kira I. Spirina ${ }^{\dagger}$ \\ Siberian Federal University \\ Krasnoyarsk, Russian Federation
}

Received 10.03.2021, received in revised form 05.04.2021, accepted 20.05.2021

\begin{abstract}
We consider the problem of determining the source function and the leading coefficient in a multidimensional semilinear parabolic equation with overdetermination conditions given on two different hypersurfaces. The existence and uniqueness theorem for the classical solution of the inverse problem in the class of smooth bounded functions is proved. A condition is found for the dependence of the upper bound of the time interval, in which there is a unique solution to the inverse problem, on the input data.
\end{abstract}

Keywords: inverse problem, overdetermination conditions, semilinear multidimensional parabolic equation, Cauchy problem, weak approximation method, input data, identification of coefficients.

Citation: S.V. Polyntseva, K.I. Spirina, The Problem of Determining of the Source Function and of the Leading Coefficient in the Many-dimensional Semilinear Parabolic Equation, J. Sib. Fed. Univ. Math. Phys., 2021, 14(4), 497-506. DOI: 10.17516/1997-1397-2021-14-4-497-506.

\section{Introduction}

The purpose of this work is to investigate the unique solvability of the problem of determining the source function and the coefficient at the second derivative in the spatial variable in a multidimensional semilinear parabolic equation with Cauchy data and overdetermination conditions, given on two different hypersurfaces. The unique solvability in classes of smooth bounded functions of various inverse problems of determining two coefficients of semilinear parabolic equations, different from the inverse problem considered in this article, was studied, for example in [1-3].

Using the overdetermination conditions, the initial inverse problem is reduced to the direct auxiliary Cauchy problem for the nonlinear loaded equation. The solvability of the direct problem is proved, for this purpose rather smooth input data and the method of weak approximation are used $[4,5]$. The solution of the original inverse problem is written out explicitly through the solution of the direct problem. On this basis, the existence and uniqueness theorem for the classical solution of the inverse problem in the class of smooth bounded functions is proved for $t^{*} \in(0, T], T>0, T-$ const. The condition for the dependence of $t^{*}$ on the constants of the sufficiently smooth input data is formulated.

\footnotetext{
*svpolyntseva@gmail.com https://orcid.org/0000-0002-8480-6612.

${ }^{\dagger}$ ki.spirina@gmail.com https://orcid.org/0000-0002-3510-7292

(c) Siberian Federal University. All rights reserved
} 


\section{Statement of the problem}

We consider in $G_{[0, T]}=\left\{(t, x, z) \mid 0 \leqslant t \leqslant T, x \in E_{n}, z \in E_{1}\right\}$ the Cauchy problem

$$
\begin{gathered}
\frac{\partial u}{\partial t}=L_{x}(u)+a(t, x) u_{z z}+\beta_{1}(t, x) u_{z}+\beta_{2}(t, x) u^{2}+b(t, x) f(t, x, z), \\
u(0, x, z)=u_{0}(x, z), \quad(x, z) \in E_{n+1} .
\end{gathered}
$$

Here $L_{x}(u)=\sum_{i, j=1}^{n} \alpha_{i j} \frac{\partial^{2} u}{\partial x_{i} \partial x_{j}}+\sum_{i=1}^{n} \alpha_{i} \frac{\partial u}{\partial x_{i}}$, the functions $u_{0}(x, z), f(t, x, z)$ are given in $E_{n+1}$ and $G_{[0, T]}$ respectively, the coefficients $\alpha_{i j}(t), \alpha_{i}(t), i, j=\overline{1, n}, \beta_{1}(t, x), \beta_{2}(t, x)$ are continuously differentiable real-valued functions of the variable $t$, and $t, x$ respectively, $0 \leqslant t \leqslant T, T>0$, $T$-const, $E_{n}$ is the $n$-dimensional Euclidean space, $n \geqslant 1, n \in \mathbf{N}$.

Let be $\alpha_{i j}(t)=\alpha_{j i}(t)$ and the relation $\sum_{i, j=1}^{n} \alpha_{i j} \xi_{i} \xi_{j}>0 \forall \xi \in E_{n} \backslash\{0\}, t \in[0, T]$ is true.

The coefficients $a(t, x), b(t, x)$ and the solution $u(t, x, z)$ of (1), (2) are unknown.

We assume that overdetermination conditions are given on two different hypersurfaces $z=d_{1}(t)$ and $z=d_{2}(t)$ :

$$
u\left(t, x, d_{1}(t)\right)=\phi(t, x), \quad u\left(t, x, d_{2}(t)\right)=\psi(t, x),
$$

where $(t, x) \in \Pi_{[0, T]}, \Pi_{[0, T]}=\left\{(t, x) \mid 0 \leqslant t \leqslant T, x \in E_{n}\right\} ; d_{1}(t), d_{2}(t)$ are continuously differentiable functions of the variable $t, d_{1}(t) \neq d_{2}(t) ; \phi(t, x), \psi(t, x)$ are given functions satisfying the matching conditions

$$
\phi(0, x)=u_{0}\left(x, d_{1}(0)\right), \quad \psi(0, x)=u_{0}\left(x, d_{2}(0)\right)
$$

where $x \in E_{n}$.

The solution of the inverse problem (1)-(3) in $G_{\left[0, t^{*}\right]}, 0<t^{*} \leqslant T$, is a triple of functions $u(t, x, z), a(t, x), b(t, x)$, that satisfies relations (1)-(3). Below we consider classical (sufficiently smooth) solutions.

\section{The transition from an inverse problem to a direct problem}

We reduce the problem (1)-(3) to some auxiliary direct problem. Let be $z=d_{1}(t), z=d_{2}(t)$ in (1) and in view of (3), we obtain

$$
\begin{aligned}
& P=\left.a(t, x) u_{z z}\right|_{z=d_{1}(t)}+b(t, x) f\left(t, x, d_{1}(t)\right), \\
& Q=\left.a(t, x) u_{z z}\right|_{z=d_{2}(t)}+b(t, x) f\left(t, x, d_{2}(t)\right),
\end{aligned}
$$

where

$$
\begin{gathered}
P=P(t, x)=F_{1}-\left.\left(\beta_{1}(t, x)+d_{1}^{\prime}(t)\right) u_{z}\right|_{z=d_{1}(t)}, \quad Q=Q(t, x)=F_{2}-\left.\left(\beta_{1}(t, x)+d_{2}^{\prime}(t)\right) u_{z}\right|_{z=d_{2}(t)}, \\
F_{1}=\phi_{t}(t, x)-L_{x}(\phi(t, x))-\beta_{2}(t, x) \phi^{2}(t, x), \quad F_{2}=\psi_{t}(t, x)-L_{x}(\psi(t, x))-\beta_{2}(t, x) \psi^{2}(t, x) .
\end{gathered}
$$


Using the Cramer's method, we find:

$$
\begin{aligned}
a(t, x) & =\frac{P f\left(t, x, d_{2}(t)\right)-Q f\left(t, x, d_{1}(t)\right)}{\left.u_{z z}\right|_{z=d_{1}(t)} f\left(t, x, d_{2}(t)\right)-\left.f\left(t, x, d_{1}(t)\right) u_{z z}\right|_{z=d_{2}(t)}}, \\
b(t, x) & =\frac{\left.Q u_{z z}\right|_{z=d_{1}(t)}-\left.P u_{z z}\right|_{z=d_{2}(t)}}{\left.u_{z z}\right|_{z=d_{1}(t)} f\left(t, x, d_{2}(t)\right)-\left.f\left(t, x, d_{1}(t)\right) u_{z z}\right|_{z=d_{2}(t)}} .
\end{aligned}
$$

We denote:

$$
\begin{gathered}
N_{1}=N_{1}(t, x)=P f\left(t, x, d_{2}(t)\right)-Q f\left(t, x, d_{1}(t)\right), \\
N_{2}=N_{2}(t, x)=\left.u_{z z}\right|_{z=d_{1}(t)} f\left(t, x, d_{2}(t)\right)-\left.f\left(t, x, d_{1}(t)\right) u_{z z}\right|_{z=d_{2}(t)}, \\
N_{3}=N_{3}(t, x)=\left.Q u_{z z}\right|_{z=d_{1}(t)}-\left.P u_{z z}\right|_{z=d_{2}(t) .}
\end{gathered}
$$

Then, substituting (5) into (1), we turn to the following problem:

$$
\begin{gathered}
u_{t}=L_{x}(u)+\frac{N_{1}}{N_{2}} u_{z z}(t, x, z)+\beta_{1}(t, x) u_{z}(t, x, z)+\beta_{2}(t, x) u^{2}(t, x, z)+\frac{N_{3}}{N_{2}} f(t, x, z), \\
u(0, x, z)=u_{0}(x, z) .
\end{gathered}
$$

We introduce the cutoff function $S_{\delta}(y) \in C^{4}\left(E_{1}\right)$, with the following properties:

$$
S_{\delta}(y) \geqslant \frac{\delta}{3}>0, S_{\delta}(y)=\left\{\begin{array}{l}
y, y \geqslant \frac{\delta}{2}, \\
\chi(y), \frac{\delta}{3}<y<\frac{\delta}{2}, \\
\frac{\delta}{3}, y \leqslant \frac{\delta}{3},
\end{array}\right.
$$

where $y \in E_{1}, \delta=$ const, $\chi(y) \in C^{4}\left(E_{1}\right)$.

We replace in (7) $N_{1}$ and $N_{2}$ by $S_{\delta_{1}}\left(N_{1}(t, x)\right), S_{\delta_{2}}\left(N_{2}(t, x)\right)$ respectively, we obtain

$$
u_{t}=L_{x}(u)+\frac{S_{\delta_{1}}\left(N_{1}(t, x)\right)}{S_{\delta_{2}}\left(N_{2}(t, x)\right)} u_{z z}+\beta_{1}(t, x) u_{z}+\beta_{2}(t, x) u^{2}+\frac{N_{3}(t, x)}{S_{\delta_{2}}\left(N_{2}(t, x)\right)} f(t, x, z) .
$$

We assume that the input data are sufficiently smooth and it has all the continuous derivatives contained in the following relation

$$
\begin{array}{r}
\left|D_{x}^{\gamma} \frac{\partial^{k}}{\partial z^{k}} \frac{\partial^{g}}{\partial t^{g}} f(t, x, z)\right|+\left|D_{x}^{\gamma} \frac{\partial^{k}}{\partial z^{k}} u_{0}(x, z)\right|+\left|D_{x}^{\gamma} \frac{\partial^{g}}{\partial t^{g}} \beta_{1}(t, x)\right|+\left|D_{x}^{\gamma} \frac{\partial^{g}}{\partial t^{g}} F_{s}(t, x)\right|+\left|\frac{d^{s_{1}}}{d t^{s_{1}}} d_{s}(t)\right| \leqslant C, \\
k=\overline{0,10-2|\gamma|},|\gamma| \leqslant 4, g=0,1, s=1,2, s_{1}=1,2 . \quad
\end{array}
$$

Here $(t, x, z) \in G_{[0, T]}, \gamma=\left(\gamma_{1}, \ldots, \gamma_{n}\right)$ is multi-index, $|\gamma|=\sum_{i=0}^{n} \gamma_{i}, D_{x}^{\gamma}=\frac{\partial^{|\gamma|}}{\partial x_{1}^{\gamma_{1}} \ldots \partial x_{n}^{\gamma_{n}}}, C$ is a constant more than one. Generally speaking, constants $C$ here and throughout are different.

Let us suppose that the following conditions are true

$$
\begin{gathered}
N_{1}(0, x)=P(0, x) f\left(0, x, d_{2}(0)\right)-Q(0, x) f\left(0, x, d_{1}(0)\right) \geqslant \delta_{1}, \\
N_{2}(0, x)=\frac{\partial^{2} u_{0}\left(x, d_{1}(0)\right)}{\partial z^{2}} f\left(0, x, d_{2}(0)\right)-\frac{\partial^{2} u_{0}\left(x, d_{2}(0)\right)}{\partial z^{2}} f\left(0, x, d_{1}(0)\right) \geqslant \delta_{2},
\end{gathered}
$$

where $(t, x) \in \Pi_{[0, T]}, \delta_{1}, \delta_{2}>0, \delta_{1}, \delta_{2}=$ const, and

$$
\begin{aligned}
& P(0, x)=\phi_{t}(0, x)-L_{x}(\phi(0, x))-\left.\left(\beta_{1}(0, x)+d_{1}^{\prime}(0)\right) u_{0 z}\right|_{z=d_{1}(0)}-\beta_{2}(0, x) \phi^{2}(0, x), \\
& Q(0, x)=\psi_{t}(0, x)-L_{x}(\psi(0, x))-\left.\left(\beta_{1}(0, x)+d_{2}^{\prime}(0)\right) u_{0 z}\right|_{z=d_{2}(0)}-\beta_{2}(0, x) \psi^{2}(0, x) .
\end{aligned}
$$

Let us prove the existence of a solution of the auxiliary direct problem (10), (8). 


\section{Solvability of the direct problem}

We apply the method of weak approximation $[4,5]$ to prove the existence of the solution of the problem $(10),(8)$. We split the problem and linearize it by a $\frac{\tau}{3}$ time shift in the nonlinear terms

$$
\begin{gathered}
u_{t}^{\tau}=3 L_{x}\left(u^{\tau}\right), \quad n \tau<t \leqslant\left(n+\frac{1}{3}\right) \tau, \\
u_{t}^{\tau}=3\left(\frac{S_{\delta_{1}}\left(N_{1}^{\tau}(t, x)\right)}{S_{\delta_{2}}\left(N_{2}^{\tau}(t, x)\right)} u_{z z}^{\tau}+\beta_{1}(t, x) u_{z}^{\tau}\right), \quad\left(n+\frac{1}{3}\right) \tau<t \leqslant\left(n+\frac{2}{3}\right) \tau, \\
u_{t}^{\tau}=3\left(\beta_{2}(t, x) u^{\tau} u^{\tau}\left(t-\frac{\tau}{3}\right)+\frac{N_{3}^{\tau}(t, x)}{S_{\delta_{2}}\left(N_{2}^{\tau}(t, x)\right)} f(t, x, z)\right), \quad\left(n+\frac{2}{3}\right) \tau<t \leqslant(n+1) \tau, \\
u^{\tau}(0, x, z)=u_{0}(x, z), \quad x \in E_{n}, z \in E_{1} .
\end{gathered}
$$

Here $n=0,1, \ldots, N-1, \tau N=T, N>0, N \in \mathbf{Z}, u^{\tau}=u^{\tau}(t)=u^{\tau}(t, x, z)$,

$$
\begin{gathered}
N_{1}^{\tau}=N_{1}^{\tau}(t, x)=P^{\tau} f\left(t, x, d_{2}(t)\right)-Q^{\tau} f\left(t, x, d_{1}(t)\right), \\
N_{2}^{\tau}=N_{2}^{\tau}(t, x)=\left.u_{z z}^{\tau}\left(t-\frac{\tau}{3}, x, d_{1}(t)\right) f\right|_{z=d_{2}(t)}-\left.f\right|_{z=d_{1}(t)} u_{z z}^{\tau}\left(t-\frac{\tau}{3}, x, d_{2}(t)\right), \\
N_{3}^{\tau}=N_{3}^{\tau}(t, x)=Q^{\tau} u_{z z}^{\tau}\left(t-\frac{\tau}{3}, x, d_{1}(t)\right)-P^{\tau} u_{z z}^{\tau}\left(t-\frac{\tau}{3}, x, d_{2}(t)\right), \\
P^{\tau}=F_{1}-\left(\beta_{1}(t, x)+d_{1}^{\prime}(t)\right) u_{z}^{\tau}\left(t-\frac{\tau}{3}, x, d_{1}(t)\right), \quad Q^{\tau}=F_{2}-\left(\beta_{1}(t, x)+d_{2}^{\prime}(t)\right) u_{z}^{\tau}\left(t-\frac{\tau}{3}, x, d_{2}(t)\right) .
\end{gathered}
$$

We introduce the notation

$$
\begin{gathered}
U^{\tau, t_{0}}(t)=\sum_{k=0}^{10} U_{k}^{\tau, t_{0}}(t), \\
U_{k}^{\tau, t_{0}}(t)=\sup _{t_{0}<\xi \leqslant t} \sup _{x \in E_{n}, z \in E_{1}}\left|\frac{\partial^{k}}{\partial z^{k}} u^{\tau}(\xi, x, z)\right|, \\
U_{k}(0)=\sup _{x \in E_{n}, z \in E_{1}}\left|\frac{\partial^{k}}{\partial z^{k}} u_{0}(x, z)\right|, \\
U_{k}^{\tau, t_{0}}\left(t_{0}\right)=\sup _{x \in E_{n}, z \in E_{1}}\left|\frac{\partial^{k}}{\partial z^{k}} u^{\tau}\left(t_{0}, x, z\right)\right|, t \in\left(t_{0},\left(n+\frac{p}{3}\right) \tau\right], \\
t_{0} \in\left[0,\left(n+\frac{p}{3}\right) \tau\right), t>t_{0}, p=1,2,3 .
\end{gathered}
$$

The functions $U_{k}^{\tau, t_{0}}(t), U_{k}^{\tau, t_{0}}\left(t_{0}\right), U_{k}(0)$ are nonnegative and non-decreasing on each half-open interval $(n \tau,(n+1) \tau]$.

Let us prove the priori estimates guaranteeing the compactness of a set of solutions $\left\{u^{\tau}(t, x, z)\right\}$ of the problem (13)-(16).

Let the half-interval $(n \tau,(n+1) \tau]$ be n-th time step, where $n=0,1, \ldots, N-1$.

We consider the zero integer step $(n=0)$.

At the first fractional step $(p=1)$, we obtain the following estimate for the solution $u^{\tau}$ of problem (13), (16), due to (11) and the maximum principle [6]

$$
\left|u^{\tau}(\xi, x, z)\right| \leqslant \sup _{x \in E_{n}, z \in E_{1}}\left|u_{0}(x, z)\right|, \quad 0<\xi \leqslant \frac{\tau}{3} .
$$


We obtain the following estimates using differentiating the equation (13), (16) with respect to $z$ from one to ten times, respectively, due to (11) and the maximum principle [6]

$$
\left|\frac{\partial^{k}}{\partial z^{k}} u^{\tau}(\xi, x, z)\right| \leqslant \sup _{x \in E_{n}, z \in E_{1}}\left|\frac{\partial^{k}}{\partial z^{k}} u_{0}(x, z)\right|, \quad k=\overline{1,10}, \quad 0<\xi \leqslant \frac{\tau}{3} .
$$

We obtain the following estimate from (20), (21) through (17), (18)

$$
U^{\tau, 0}(t) \leqslant U(0), \quad 0<t \leqslant \frac{\tau}{3} .
$$

At the second fractional step $(p=2)$, we obtain the following estimate for the solution of equation (14) with initial data $u^{\tau}\left(\frac{\tau}{3}, x, z\right)$ due to (11), (9) and the maximum principle [6]

$$
U^{\tau, \frac{\tau}{3}}(t) \leqslant U^{\tau, \frac{\tau}{3}}\left(\frac{\tau}{3}\right), \quad \frac{\tau}{3}<t \leqslant \frac{2 \tau}{3} .
$$

Collectively, on the first and second fractional steps, due to (22), (23) we get

$$
U^{\tau, 0}(t) \leqslant U(0), \quad 0<t \leqslant \frac{2 \tau}{3} .
$$

At the third fractional step $(p=3)$, integrating the equation (15) with $t \in\left(\frac{2 \tau}{3}, \xi\right], \frac{2 \tau}{3}<\xi \leqslant \tau$, we receive the equality

$$
u^{\tau}(\xi)=u^{\tau}\left(\frac{2 \tau}{3}\right)+3 \int_{\frac{2 \tau}{3}}^{\xi}\left(\beta_{2}(\eta, x) u^{\tau}(\eta) u^{\tau}\left(\eta-\frac{\tau}{3}, x, z\right)+\frac{N_{3}^{\tau}(\eta, x)}{S_{\delta_{2}}\left(N_{2}^{\tau}(\eta, x)\right)} f(\eta, x, z)\right) d \eta .
$$

The last relation implies the inequality

$$
\left|u^{\tau}(\xi)\right| \leqslant\left|u^{\tau}\left(\frac{2 \tau}{3}\right)\right|+3 \int_{\frac{2 \tau}{3}}^{\xi}\left(\left|\beta_{2}(\eta, x)\right| u^{\tau}(\eta)|| u^{\tau}\left(\eta-\frac{\tau}{3}\right)\left|+\frac{\left|N_{3}^{\tau}(\eta, x)\right|}{\left|S_{\delta_{2}}\left(N_{2}^{\tau}(\eta, x)\right)\right|}\right| f(\eta, x, z) \mid\right) d \eta,
$$

where $\frac{2 \tau}{3}<\xi \leqslant t \leqslant \tau$.

Since this inequality holds for all $x, z$ we replace the functions of the integral terms by their exact upper bounds with respect to $x \in E_{n}, z \in E_{1}$, and then replace the function $\left|u^{\tau}\right|$, on the left-hand side of the inequality by $\sup _{x \in E_{n} z \in E_{1}}\left|u^{\tau}\right|$ considering (17)-(19) we obtain

$$
\begin{array}{r}
U_{0}^{\tau, \frac{2 \tau}{3}}(t) \leqslant U_{0}^{\tau, \frac{2 \tau}{3}}\left(\frac{2 \tau}{3}\right)+C \int_{\frac{2 \tau}{3}}^{t}\left(U_{0}^{\tau, \frac{2 \tau}{3}}(\eta) U_{0}^{\tau, \frac{2 \tau}{3}}\left(\eta-\frac{\tau}{3}\right)+U_{2}^{\tau, \frac{2 \tau}{3}}\left(\eta-\frac{\tau}{3}\right)+\right. \\
\left.+U_{2}^{\tau, \frac{2 \tau}{3}}\left(\eta-\frac{\tau}{3}\right) U_{1}^{\tau, \frac{2 \tau}{3}}\left(\eta-\frac{\tau}{3}\right)\right) d \eta
\end{array}
$$

Further, in the same way, differentiating equations (15) with respect to $z$ from one to 10 times, similarly to the second fractional step, we get

$$
\begin{aligned}
U_{k}^{\tau, \frac{2 \tau}{3}}(t) \leqslant U_{k}^{\tau, \frac{2 \tau}{3}}\left(\frac{2 \tau}{3}\right)+C \int_{\frac{2 \tau}{3}}^{t} \sum_{q=0}^{k}\left(U_{k-q}^{\tau, \frac{2 \tau}{3}}(\eta) U_{q}^{\tau, \frac{2 \tau}{3}}\left(\eta-\frac{\tau}{3}\right)+U_{2}^{\tau, \frac{2 \tau}{3}}\left(\eta-\frac{\tau}{3}\right)+\right. \\
\left.+U_{2}^{\tau, \frac{2 \tau}{3}}\left(\eta-\frac{\tau}{3}\right) U_{1}^{\tau, \frac{2 \tau}{3}}\left(\eta-\frac{\tau}{3}\right)\right) d \eta, \quad k=\overline{1,10} . \\
-501-
\end{aligned}
$$


Adding (25) and (26), by virtue of (17) we receive

$$
\begin{array}{r}
U^{\tau, \frac{2 \tau}{3}}(t) \leqslant U^{\tau, \frac{2 \tau}{3}}\left(\frac{2 \tau}{3}\right)+C \int_{\frac{2 \tau}{3}}^{t}\left(U^{\tau, \frac{2 \tau}{3}}(\eta) U^{\tau, \frac{2 \tau}{3}}\left(\eta-\frac{\tau}{3}\right)+U^{\tau, \frac{2 \tau}{3}}\left(\eta-\frac{\tau}{3}\right)+\right. \\
\left.+U^{\tau, \frac{2 \tau}{3}}\left(\eta-\frac{\tau}{3}\right) U^{\tau, \frac{2 \tau}{3}}\left(\eta-\frac{\tau}{3}\right)\right) d \eta
\end{array}
$$

or

$$
\begin{array}{r}
U^{\tau, \frac{2 \tau}{3}}(t) \leqslant U^{\tau, \frac{2 \tau}{3}}\left(\frac{2 \tau}{3}\right)+C \int_{\frac{2 \tau}{3}}^{t}\left(U^{\tau, \frac{2 \tau}{3}}(\eta) U^{\tau, \frac{2 \tau}{3}}\left(\frac{2 \tau}{3}\right)+U^{\tau, \frac{2 \tau}{3}}\left(\frac{2 \tau}{3}\right)+\right. \\
\left.+U^{\tau, \frac{2 \tau}{3}}\left(\frac{2 \tau}{3}\right) U^{\tau, \frac{2 \tau}{3}}\left(\frac{2 \tau}{3}\right)\right) d \eta
\end{array}
$$

where $C \geqslant 1$-constant, independent of $\tau$.

To the last inequality we apply the Gronwall lemma [7], then

$$
U^{\tau, \frac{2 \tau}{3}}(t) \leqslant\left(U^{\tau, \frac{2 \tau}{3}}\left(\frac{2 \tau}{3}\right)+1\right) e^{2 C \tau\left(U^{\tau, \frac{2 \tau}{3}}\left(\frac{2 \tau}{3}\right)+1\right)}-1, \quad \frac{2 \tau}{3}<t \leqslant \tau .
$$

Consequently, from (24) and last inequality at the zero whole step the following estimate holds

$$
U^{\tau, 0}(t) \leqslant(U(0)+1) e^{2 C \tau(U(0)+1)}-1, \quad 0<t \leqslant \tau .
$$

Repeating similar arguments at the first whole step, we obtain

$$
U^{\tau, \tau}(t) \leqslant\left(U^{\tau, \tau}(\tau)+1\right) e^{2\left(U^{\tau, \tau}(\tau)+1\right) C \tau}-1, \quad \tau<t \leqslant 2 \tau .
$$

Assuming that $\tau$ is sufficiently small and the inequality $e^{2(U(0)+1) C \tau} \leqslant 2$ holds, at the zero and first whole steps we get

$$
U^{\tau, 0}(t) \leqslant(U(0)+1) e^{6(U(0)+1) C \tau}-1, \quad 0<t \leqslant 2 \tau .
$$

Analogous reasoning, at the $n$-th whole step $(n<N)$ we obtain

$$
U^{\tau, n \tau}(t) \leqslant\left(U^{\tau, n \tau}(n \tau)+1\right) e^{2 C \tau\left(U^{\tau, n \tau}(n \tau)+1\right)}-1, \quad n \tau<t \leqslant(n+1) \tau .
$$

Consequently, at $n$ whole steps, we getting

$$
U^{\tau, 0}(t) \leqslant(U(0)+1) e^{2(2 n+1)(U(0)+1) C \tau}-1, \quad 0<t \leqslant(n+1) \tau .
$$

Hence, following estimate is true

$$
U^{\tau, 0}(t) \leqslant(U(0)+1) e^{2(U(0)+1) C t_{*}}-1, \quad 0<t \leqslant t_{*},
$$

where $t_{*}$ satisfies the inequality

$$
e^{2(U(0)+1) C t_{*}} \leqslant 2
$$

Here $U(0)=\sum_{k=0}^{10} \sup _{x \in E_{n}, z \in E_{1}}\left|\frac{\partial^{k}}{\partial z^{k}} u_{0}(x, z)\right|, C$ is constant depends of $C, \delta_{1}, \delta_{2}$ from (11), (12).

And, therefore, taking into account the notation (17), (18) uniformly with respect to $\tau$

$$
\left|\frac{\partial^{k}}{\partial z^{k}} u^{\tau}(t, x, z)\right| \leqslant C, \quad k=\overline{0,10}, \quad(t, x, z) \in G_{\left[0, t_{*}\right]} .
$$


After differentiating problem (13)-(16) with respect to $x_{i}, x_{j}, x_{l}$ and $x_{m}$, we obtain equations that can be regarded as linear with coefficients uniformly bounded in $\tau$. Arguing by analogy and considering (28), we obtain estimates uniformly with respect to $\tau$

$$
\left|D_{x}^{\gamma} \frac{\partial^{k}}{\partial z^{k}} u^{\tau}(t, x, z)\right| \leqslant C, \quad k=\overline{0,10-2|\gamma|}, \quad|\gamma| \leqslant 4, \quad(t, x, z) \in G_{\left[0, t_{*}\right]} .
$$

We obtain from (29) and (13)-(16) uniformly with respect to $\tau$

$$
\left|u_{t}^{\tau}(t, x, z)\right| \leqslant C, \quad(t, x, z) \in G_{\left[0, t_{*}\right]} .
$$

We differentiate equations (13)-(16) once with respect to $z$. By (29), the right-hand side of the equations obtained is uniformly bounded in $\tau$, and consequently the left-hand side is also uniformly bounded in $\tau$

$$
\left|u_{t z}^{\tau}(t, x, z)\right| \leqslant C, \quad(t, x, z) \in G_{\left[0, t_{*}\right]} .
$$

By analogy, uniformly with respect to $\tau$

$$
\left|\frac{\partial^{k}}{\partial z^{k}} D_{x}^{\lambda} u_{t}^{\tau}(t, x, z)\right| \leqslant C, \quad k=\overline{0,4}, \quad|\lambda| \leqslant 2, \quad(t, x, z) \in G_{\left[0, t_{*}\right]} .
$$

Thus, the following estimate holds uniformly with respect to $\tau$ for $(t, x, z) \in G_{\left[0, t_{*}\right]}$

$$
\begin{array}{r}
\left|\frac{\partial}{\partial t} \frac{\partial^{k}}{\partial z^{k}} D_{x}^{\lambda} u^{\tau}(t, x, z)\right|+\left|\frac{\partial}{\partial x_{i}} \frac{\partial^{k}}{\partial z^{k}} D_{x}^{\lambda} u^{\tau}(t, x, z)\right|+\left|\frac{\partial}{\partial z} \frac{\partial^{k}}{\partial z^{k}} D_{x}^{\lambda} u^{\tau}(t, x, z)\right| \\
k=\overline{0,4}, \quad|\lambda| \leqslant 2 .
\end{array}
$$

The estimate (29) implies the uniform boundedness in $\tau$ of the family $\left\{D_{x}^{\gamma} \frac{\partial^{k}}{\partial z^{k}} u^{\tau}\right\}$ in $G_{\left[0, t_{*}\right]}$, and from (29), (30) their equicontinuity in $t, x$ and $z$ is equicontinuous in $G_{\left[0, t_{*}\right]}$. Therefore, for any fixed $\gamma, k,|\gamma| \leqslant 2, k=\overline{0,4}$, by the Arzela theorem [8] the set $\left\{D_{x}^{\gamma} \frac{\partial^{k}}{\partial z^{k}} u^{\tau}\right\}$ is compact in $C\left(G_{\left[0, t_{*}\right]}^{M}\right), M>0$ is an integer, $G_{\left[0, t_{*}\right]}^{M}=\{(t, x, z)|t \in[0, T]| x,|\leqslant M| z \mid, \leqslant M\}$.

In a diagonal way, we choose a subsequence $\left\{u^{\tau}\right\}$ (we do not change the notation) converging together with the corresponding derivatives with respect to $x$ and $z$ to some function $u$ in $G_{\left[0, t_{*}\right]}$, and uniformly in each $G_{\left[0, t_{*}\right]}^{M}$. The function $u$ is continuous, has derivatives of the corresponding order in $x$ and $z$ that are continuous in $G_{\left[0, t_{*}\right]}$, and satisfies the initial data (2) and inequality

$$
\left|D_{x}^{\beta} \frac{\partial^{k}}{\partial z^{k}} u(t, x, z)\right| \leqslant C, \quad k=\overline{0,4}, \quad|\beta| \leqslant 2,(t, x, z) \in G_{\left[0, t_{*}\right]} .
$$

Since $D_{x}^{\gamma} \frac{\partial^{k}}{\partial z^{k}} u^{\tau} \underset{\tau \rightarrow 0}{\rightrightarrows} D_{x}^{\gamma} \frac{\partial^{k}}{\partial z^{k}} u$ on $G_{\left[0, t_{*}\right]}^{M} \forall M>0,|\gamma| \leqslant 2, k=\overline{0,4}$ and the inequality (31) is satisfied, then we can prove that the proof is similar to the proof of Theorem 2.4.1 (see Sec. 2.4. One theorem of the weak approximation method [4]) that the function $u$ is a solution of the problem $(10),(8)$ in $G_{\left[0, t_{*}\right]}^{M}$ for any fixed $M$, and since $M$ is arbitrary, then also in $G_{\left[0, t_{*}\right]}$.

The function $u(t, x, z)$ belongs to the class

$$
\begin{array}{r}
C_{t, x, z}^{1,2,4}\left(G_{\left[0, t_{*}\right]}\right)=\left\{f_{1}(t, x, z) \mid \frac{\partial^{g}}{\partial t^{g}} f_{1} \in C\left(G_{\left[0, t_{*}\right]}\right)\right), D_{x}^{\beta} \frac{\partial^{k}}{\partial z^{k}} f_{1} \in C\left(G_{\left[0, t_{*}\right]}\right), \\
|\beta| \leqslant 2, k=\overline{0,4}, g=0,1\} .
\end{array}
$$


The estimate (31) is true. In order to prove the existence of a solution of problem (7), (8), it is necessary to remove the cutoff functions in equation (10). For this, we prove that for $(t, x) \in \Pi_{\left[0, t^{*}\right]}$,

$$
N_{1}(t, x) \geqslant \frac{\delta_{1}}{2}, \quad N_{2}(t, x) \geqslant \frac{\delta_{2}}{2}
$$

We differentiate the expressions for $N_{1}(t, x), N_{2}(t, x)\left(N_{1}(t, x), N_{2}(t, x)\right.$ in (6))) with respect to $t$,

$$
\begin{gathered}
M_{1}(t, x)=\left(N_{1}(t, x)\right)_{t}^{\prime}=P_{t}^{\prime} f\left(t, x, d_{2}(t)\right)+P\left(f_{t}^{\prime}\left(t, x, d_{2}(t)\right)+f_{z}^{\prime}\left(t, x, d_{2}(t)\right) d_{2}^{\prime}(t)\right)- \\
\quad-Q_{t}^{\prime} f\left(t, x, d_{1}(t)\right)-Q\left(f_{t}^{\prime}\left(t, x, d_{1}(t)\right)+f_{z}^{\prime}\left(t, x, d_{1}(t)\right) d_{1}^{\prime}(t)\right) \\
M_{2}(t, x)=\left(N_{2}(t, x)\right)_{t}^{\prime}=\left(u_{z z t}\left(t, x, d_{1}(t)\right)+u_{z z z}\left(t, x, d_{1}(t)\right) d_{1}^{\prime}(t)\right) f\left(t, x, d_{2}(t)\right)+ \\
+u_{z z}\left(t, x, d_{1}(t)\right)\left(f_{t}^{\prime}\left(t, x, d_{2}(t)\right)+f_{z}^{\prime}\left(t, x, d_{2}(t)\right) d_{2}^{\prime}(t)\right)-\left(f_{t}^{\prime}\left(t, x, d_{1}(t)\right)+\right. \\
\left.+f_{z}^{\prime}\left(t, x, d_{1}(t)\right) d_{1}^{\prime}(t)\right) u_{z z}\left(t, x, d_{2}(t)\right)-f\left(t, x, d_{1}(t)\right)\left(u_{z z}\left(t, x, d_{2}(t)\right)\right)_{t}^{\prime}
\end{gathered}
$$

where

$$
\begin{gathered}
P_{t}^{\prime}=\phi_{t t}-L_{x t}(\phi(t, x))-\beta_{1 t}(t, x) u_{z}\left(t, x, d_{1}(t)\right)-\beta_{1}(t, x)\left(u_{z t}\left(t, x, d_{1}(t)\right)+\right. \\
\left.+u_{z z}\left(t, x, d_{1}(t)\right) d_{1}^{\prime}(t)\right)-\beta_{2 t}(t, x) \phi^{2}(t, x)-2 \beta_{2}(t, x) \phi(t, x) \phi_{t}(t, x)-\left(u_{z t}\left(t, x, d_{1}(t)\right)+\right. \\
\left.+u_{z z}\left(t, x, d_{1}(t)\right) d_{1}^{\prime}(t)\right) d_{1}^{\prime}(t)-u_{z}\left(t, x, d_{1}(t)\right) d_{1}^{\prime \prime}(t), \\
Q_{t}^{\prime}=\psi_{t t}-L_{x t}(\psi(t, x))-\beta_{1 t}(t, x) u_{z}\left(t, x, d_{2}(t)\right)-\beta_{1}(t, x)\left(u_{z t}\left(t, x, d_{2}(t)\right)+\right. \\
\left.+u_{z z}\left(t, x, d_{2}(t)\right) d_{2}^{\prime}(t)\right)-\beta_{2 t}(t, x) \psi^{2}(t, x)-2 \beta_{2}(t, x) \psi(t, x) \psi_{t}(t, x)-\left(u_{z t}\left(t, x, d_{2}(t)\right)+\right. \\
\left.+u_{z z}\left(t, x, d_{2}(t)\right) d_{2}^{\prime}(t)\right) d_{2}^{\prime}(t)-u_{z}\left(t, x, d_{2}(t)\right) d_{2}^{\prime \prime}(t), \\
L_{x t}(\phi(t, x))=\sum_{i, j=1}^{n}\left(\left(\alpha_{i j}\right)^{\prime} \frac{\partial^{2} \phi}{\partial x_{i} \partial x_{j}}+\alpha_{i j} \frac{\partial \phi}{\partial x_{i} \partial x_{j} \partial t}\right)+\sum_{i=1}^{n}\left(\left(\alpha_{i}\right)^{\prime} \frac{\partial \phi}{\partial x_{i}}+\alpha_{i} \frac{\partial^{2} \phi}{\partial x_{i} \partial t}\right), \\
L_{x t}(\psi(t, x))=\sum_{i, j=1}^{n}\left(\left(\alpha_{i j}\right)^{\prime} \frac{\partial^{2} \psi}{\partial x_{i} \partial x_{j}}+\alpha_{i j} \frac{\partial \psi}{\partial x_{i} \partial x_{j} \partial t}\right)+\sum_{i=1}^{n}\left(\left(\alpha_{i}\right)^{\prime} \frac{\partial \psi}{\partial x_{i}}+\alpha_{i} \frac{\partial^{2} \psi}{\partial x_{i} \partial t}\right) .
\end{gathered}
$$

By virtue of (11), (31)

$$
\left|M_{1}(t, x)\right| \leqslant K_{1}, \quad\left|M_{2}(t, x)\right| \leqslant K_{2},
$$

here $K_{1}, K_{2}$ are constants depending on $\delta_{1}, \delta_{2}, C$.

We integrate expressions (33) with respect to $t$ in the range from 0 to $t$, we obtain

$$
N_{1}(t, x)=N_{1}(0, x)+\int_{0}^{t} M_{1}(\eta, x) d \eta, \quad N_{2}(t, x)=N_{2}(0, x)+\int_{0}^{t} M_{2}(\eta, x) d \eta .
$$

By virtue of (12), (34) $N_{1}(t, x) \geqslant \delta_{1}-K_{1} t, N_{2}(t, x) \geqslant \delta_{2}-K_{2} t$

$$
N_{1}(t, x) \geqslant \frac{\delta_{1}}{2}, \quad N_{2}(t, x) \geqslant \frac{\delta_{2}}{2}, \quad t \in\left[0, t^{*}\right] .
$$

By the definition of the cutoff function (9) and (35), we obtain $S_{\delta_{1}}\left(N_{1}(t, x)\right)=N_{1}(t, x)$, $S_{\delta_{2}}\left(N_{2}(t, x)\right)=N_{2}(t, x)$ with $t \in\left[0, t^{*}\right]$, when $t^{*}=\min \left(t_{*}, \frac{\delta_{1}}{2 K_{1}}, \frac{\delta_{2}}{2 K_{2}}\right)$. 
Thus, in equation (10), the cutoffs are removed. The function $u(t, x, z)$ satisfies equation (7).

The coefficients $a(t, x)$ and $b(t, x)$ can be written in the form (5).

Thus, we have proved the existence of a solution $u(t, x, z)$ of the direct problem $(7),(8)$ in the class $C_{t, x, z}^{1,2,4}\left(G_{\left[0, t^{*}\right]}\right)$. It is proved

Theorem 1. Let conditions (9), (11), (12) are satisfied. Then there exists a solution $u(t, x, z)$ of the problem (7), (8) in the class $C_{t, x, z}^{1,2,4}\left(G_{\left[0, t^{*}\right]}\right)$ satisfying (31). The constant $t^{*}=\min \left(t_{*}, \frac{\delta_{1}}{2 K_{1}}, \frac{\delta_{2}}{2 K_{2}}\right)$, where $t_{*}$ satisfies the inequality $(27)$, the constants $K_{1}, K_{2}$ depend on $C, \delta_{1}, \delta_{2}$, from the relations (11), (12).

\section{The existence and uniqueness of a classical solution of the inverse problem}

Let us prove that the triple of functions $u(t, x, z), a(t, x), b(t, x)$ are the solution of the inverse problem (1)-(3), where $a(t, x)$ and $b(t, x)$ are defined in (5). Since $u(t, x, z)$ is the solution of the direct problem (7), (8), substituting $u(t, x, z), a(t, x), b(t, x)$ in (1), we obtain the correct identity.

According to (11), (31) from (5), (7), we obtain that the triple of functions $u(t, x, z), a(t, x)$, $b(t, x)$ belongs to the class

$$
\begin{array}{r}
Z\left(t^{*}\right)=\left\{u(t, x, z), a(t, x), b(t, x) \mid u \in C_{t, x, z}^{1,2,4}\left(G_{\left[0, t^{*}\right]}\right),\right. \\
\left.a(t, x), b(t, x) \in C_{t, x}^{0,2}\left(\Pi_{\left[0, t^{*}\right]}\right)\right\},
\end{array}
$$

and satisfies the inequalities

$$
\begin{gathered}
\sum_{|\beta| \leqslant 2} \sum_{k=0}^{4}\left|D_{x}^{\beta} \frac{\partial^{k}}{\partial z^{k}} u(t, x, z)\right| \leqslant C, \quad(t, x, z) \in G_{\left[0, t^{*}\right]}, \\
\sum_{|\beta| \leqslant 2}\left|D_{x}^{\beta} a(t, x)\right|+\sum_{|\beta| \leqslant 2}\left|D_{x}^{\beta} b(t, x)\right| \leqslant C, \quad(t, x) \in \Pi_{\left[0, t^{*}\right]} .
\end{gathered}
$$

The class $C_{t, x, z}^{1,2,4}\left(G_{\left[0, t^{*}\right]}\right)$ is defined in $(32)$, and

$$
C_{t, x}^{0,2}\left(\Pi_{\left[0, t^{*}\right]}\right)=\left\{a_{1}(t, x)\left|D_{x}^{\beta} a_{1}(t, x) \in C\left(\Pi_{\left[0, t^{*}\right]}\right),\right| \beta \mid \leqslant 2\right\} .
$$

Using conditions (4) and equation (1), we can prove that the overdetermination conditions (3) are satisfied.

The existence in the class $Z\left(t^{*}\right)$ of the solution $u(t, x, z), a(t, x), b(t, x)$ of the problem (1)-(3) satisfying relations (1)-(3) is proved.

The uniqueness of the solution to problem (1)-(3) is proved by a standard method: the difference between the two solution to problem (1)-(3) that obey $(36),(37)$ is shown to vanish.

Thus, it is proved

Theorem 2. Let us conditions (4), (11), (12) are satisfied. Then there exists a unique solution $u(t, x, z), a(t, x), b(t, x)$ of problem (1)-(3) in the class $Z\left(t^{*}\right)$ satisfying relations (36), (37). The constant $t^{*}=\min \left(t_{*}, \frac{\delta_{1}}{2 K_{1}}, \frac{\delta_{2}}{2 K_{2}}\right)$, where $t_{*}$ satisfies the inequality (27), the constants $K_{1}, K_{2}$ of the temple from $C, \delta_{1}, \delta_{2}$, from relations (11), (12). 


\title{
References
}

[1] Yu.Ya.Belov, I.V.Frolenkov, On the problem of identification two coefficients of a parabolic semilinear equation, Vestnik KrasGU, 1(2004), 140-149 (in Russian).

[2] Yu.Ya.Belov, I.V.Frolenkov, Coefficient Identification Problems for Semilinear Parabolic Equations, Doklady Mathematics, 72(2005) no. 2, 737-739.

[3] Yu.Ya.Belov, I.V.Frolenkov, On the problem of identifying two coefficients of a parabolic semilinear equation with overdetermination conditions defined on a smooth curve, Computational technologies, 11(2006), part 1, 46-54 (in Russian).

[4] Yu.Ya.Belov, S.A.Cantor, The method of weak approximation, Krasnoyarsk, KrasGU, 1999.

[5] N.N.Yanenko, Method of fractional steps for solving multidimensional problems of mathematical physics, Novosibirsk, 1967.

[6] A.M.Il'in, A.S.Kalashnikov, O.A.Oleynik, Linear second-order equations of parabolic type, $U M N, \mathbf{1 7}(1962), 3-146$.

[7] B.M.Rozhdestvenskiy, N.N.Yanenko, Systems of quasilinear equations, Moscow, Nauka, 1978.

[8] L. V.Kantorovich, G.P.Akilov, Functional Analysis. 2nd Edition, Moscow, Nauka, 1977.

\section{Задача определения функции источника и старшего коэффициента в полулинейном многомерном параболическом уравнении}

\author{
Светлана В. Полынцева \\ Кира И. Спирина \\ Сибирский федеральный университет \\ Красноярск, Российская Федерация
}

\begin{abstract}
Аннотация. Рассматривается задача определения функции источника и коэффициента при второй производной по пространственной переменной в многомерном полулинейном параболическом уравнении с условиями переопределения, заданными на двух различных гиперповерхностях. Доказана теорема существования и единственности классического решения обратной задачи в классе гладких ограниченных функций. Найдено условие зависимости верхней границы временного отрезка, в котором существует и единственно решение обратной задачи, от входных данных.

Ключевые слова: обратная задача, условия переопределения, полулинейное многомерное параболическое уравнение, задача Коши, метод слабой аппроксимации, входные данные, определение коэффициентов.
\end{abstract}

